\title{
Integration von Gender und Diversity-Aspekten in die Informatik-Lehre - Ausgewählte Beispiele der FH Erfurt und der TU Ilmenau
}

\author{
Kristin Probstmeyer ${ }^{1}$, Gabriele Schade ${ }^{2}$ \\ TU Ilmenau ${ }^{1}$ \\ FH Erfurt ${ }^{2}$
}

1

\section{Informatik und Gender}

Die Anbindung von Gender und Diversity im Kontext der Informatik-Lehre erschließt sich für Lehrende nicht immer auf den ersten Blick und erweist sich bei näherer Betrachtung als äußerst komplex. So lassen sich zunächst zwei zentrale Aspekte ausmachen:

Zum einen geht es, dem politischen Gendermainstreaming-Auftrages entsprechend, um die gleichberechtigte Teilhabe von Frauen und Männern in den Studien- und Berufsfeldern der Informatik. Mit Zugangsvoraussetzungen an deutschen Hochschulen, die zur Aufnahme eines (Informatik)-Studiums unabhängig von Geschlecht, Alter, Ethnizität, sozialem Status etc. motivieren, wurden bereits wichtige Grundlagen auf struktureller Ebene geschaffen. Ein Blick auf das Geschlechterverhältnis in der Informatik zeigt, dass sich in den letzten Jahren die Anzahl weiblicher Informatik-Studierender in Deutschland - zugegebenermaßen auf niedrigem Niveau - nahezu verdoppelt hat: von rund 6.200 Studentinnen im Jahr 2010 auf rund 12.000 Studentinnen im Jahr 2012. Trotz dieser positiven Tendenz liegt der Frauenanteil jedoch mit $18 \%$ immer noch deutlich unter dem der männlichen Informatik-Studierenden (VDI-monitorING, 2013). Als Gründe werden unter anderem nach wie vor gesellschaftlich vorherrschende Geschlechterrollenstereotype und eine männlich konnotierte Fachkultur genannt (Berszinski et al., 2002; Derboven und Winker, 2010; Engler, 1993; Münst, 2002; Ripke und Siegeris, 2012; Roloff, 1988; Schinzel, 2012).

Zum anderen rückt auch die inhaltliche Ebene zunehmend in den Fokus. So prägen aktuelle Erkenntnisse der Technikdidaktik sowie der hochschuldidaktischen Gender- und Diversityforschung maßgeblich die Vorstellung von einer zeitgemäßen guten Hochschullehre, zu deren Qualitätsmerkmalen auch die Berücksichtigung der verschiedenen Lebenswirklichkeiten der Studierenden zählt. Neben dem Gespür für und dem Entgegenwirken von Ungleichheiten aufgrund sozialer Rollen, gilt es in Lehrveranstaltungen die individuellen Interessen und Lernbedürfnisse von Studierenden zu ermitteln und zu bedienen (Götsch, 2011; GrünewaldHuber und von Gunten, 2009; Jansen-Schulz und van Riesen, 2009; Liebig, RosenkranzFallegger und Meyerhofer, 2009; Becker, Jansen-Schulz, Kortendiek und Schäfer, 2006; Rosser, 1995). 


\section{Gender- und Diversity-Aspekte in der Informatik-Lehre und Software-Ergonomie/Usability}

Neben den formalen Rahmenbedingungen und der gender- und diversitysensiblen Gestaltung der Lehr- und Lernbedingungen, gewinnt insbesondere die inhaltliche Thematisierung von Gender- und Diversity-Aspekten in der Informatik-Lehre an Bedeutung. So werden Hochschulen zunehmend in die Verantwortung genommen, Fachkräfte auszubilden, die sowohl bei der Technikentwicklung und Technikgestaltung als auch im Hinblick auf Techniknutzung gender- und diversitysensibel agieren. Mit der Prämisse, dass Technik von Menschen für Menschen entwickelt wird, sollen beispielsweise interdisziplinäre Bezüge (soziale, wirtschaftliche und rechtliche Komponenten) eine stärkere Gewichtung erhalten.

Unter dem Schlagwort „Benutzerorientierte Entwicklung“ sind vor allem in der SoftwareErgonomie und der Usability zunehmend Kenntnisse über Bedürfnisse und Wünsche der jeweiligen Zielgruppen gefragt. Diese Anforderungen des Marktes führen dazu, dass Informatik-Studierende frühzeitig an Usability und Spezifika von Nutzergruppen herangeführt werden (u. a. Beier und von Gizycki, 2002).

Alles das wäre doch eine gute Nachricht für „Gender und Informatik“, doch leider ist die Berücksichtigung von Gender- und Diversity-Aspekten in die Softwareentwicklung, -gestaltung und -nutzung nicht nur das (marketingdeterminierte) Einbringen von Zielgruppenbedürfnissen, das häufig auch noch geschlechtsspezifische Stereotypen verstärkt, sondern vor allem das Einbringen der Genderperspektive in den gesamten Software-EngineeringProzess (Erharter, 2012).

Mit Blick auf die Lehrenden der Informatik zeigt sich dann, dass trotz dieser notwendigen und zielführenden Intentionen und mitunter auch Aufgeschlossenheit gegenüber dieser Thematik, sich eine praktische Umsetzung oft schwierig erweist. Gründe hierfür sind unter anderem ein straffer Lehrplan, knappe Personal- und Zeitressourcen oder mangelnde GenderDiversity-Kenntnisse von Informatik-Lehrenden bzw. fehlende Konzepte zur Einbettung von Gender- und Diversity-Themen in eigene Fachinhalte (Augustin und Probstmeyer, 2013; Döring und Probstmeyer, 2013). Im vorliegenden Beitrag werden daher ausgewählte Genderund Diversitytools vorgestellt.

Gemeinsam mit den anderen Thüringer Hochschulen kooperieren die Fachhochschule Erfurt und die Technische Universität Ilmenau im Forschungsprojekt „GeniaL - Gender in der akademischen Lehre“ (Projektlaufzeit: 2009 bis 2012) sowie dem „Thüringer Kompetenznetzwerk Gleichstellung“ (TKG - Projektlaufzeit: 2013-2015). Beide Thüringer Verbundvorhaben wurden maßgeblich von der Landeskonferenz der Gleichstellungsbeauftragten an Thüringer Hochschulen (LaKoG) initiiert und durch das Thüringer Ministerium für Bildung, Wissenschaft und Kultur (TMBWK) gefördert. Erhebung, Vernetzung und (Weiter)Entwicklung von Gender-Diversity-Aktivitäten der Thüringer Hochschulen zählen zu den Hauptaufgaben der Verbundarbeit.

Das Beratungs- und Forschungskonzept des Projekts GeniaL zur Gestaltung einer gendersensiblen Lehre an den Thüringer Hochschulen, mit Maßnahmen und ausgewählten Ergebnissen 
sind im Forschungsbericht publiziert (siehe Döring, Augustin und Probstmeyer, 2013). Einige Beispiele zu den Lehr- und Forschungsaktivitäten im Bereich der Informatik werden im Folgenden vorgestellt.

\subsection{Gender- und Diversity-Aspekte in der (Informatik-)Lehre aus Sicht der Studierenden und Lehrenden}

Eine im Projekt GeniaL durchgeführte Befragung mit rund 1.500 Studierenden (darunter 142 Informatikstudierende, $14 \%$ weiblich und $86 \%$ männlich) der Thüringer Hochschulen ergab, dass, unabhängig von der jeweiligen Fächergruppe, Studierende

- in ihren Lehrinhalten kaum Genderbezüge sehen,

- Wissensdefizite im Hinblick auf Genderwissen aufweisen,

- Gleichstellungsthemen eher desinteressiert gegenüberstehen.

Des Weiteren zeigte sich in einer qualitativen Studie, in der 21 Interviews mit Lehrenden und 31 Unterrichtsbeobachtungen durchgeführt wurden, dass Lehrende, unabhängig ihrer Fächerkultur

- Hochschullehre als geschlechtsneutral definieren

- sich dennoch geschlechtsdifferenzierend verhalten und kaum reflexive GenderKompetenzen besitzen

- Genderthemen vorwiegend als „frauenspezifische Themen“ reflektieren

Diese Ergebnisse bestärkten die Notwendigkeit von gezielten Beratungs- und Weiterbildungsangeboten im Bereich der gender- und diversitysensiblen Hochschuldidaktik an den Thüringer Hochschulen. So wurde eine Gender-Diversity-Toolbox entwickelt, die Handlungsempfehlungen und konkrete Gestaltungsbeispiele enthält, von Lehrenden auch ohne spezifische Vorkenntnisse zu handhaben ist und auch von Studierenden positiv bewertet wird (Döring et al. 2013; John, Probstmeyer, Saarmann und Schade, 2012).

\subsection{Gender-Diversity-Toolbox für (Informatik-)Lehrende}

Die Beispiele der Gender-Diversity-Toolbox eignen sich besonders gut für Lehrveranstaltungen des Grundlagenstudiums und zielen darauf, Gender- und Diversity-Aspekte in den bestehenden Lehrstoff einzugliedern (siehe Prinzip des Integrativen Genderings von JansenSchulz und van Riesen, 2009). Wie in Abbildung 1 veranschaulicht, wurden für die GenderDiversity-Toolbox insgesamt acht Dimensionen definiert, die sich an den Gestaltungskriterien einer guten und gender-/diversitysensiblen Lehre orientieren. 


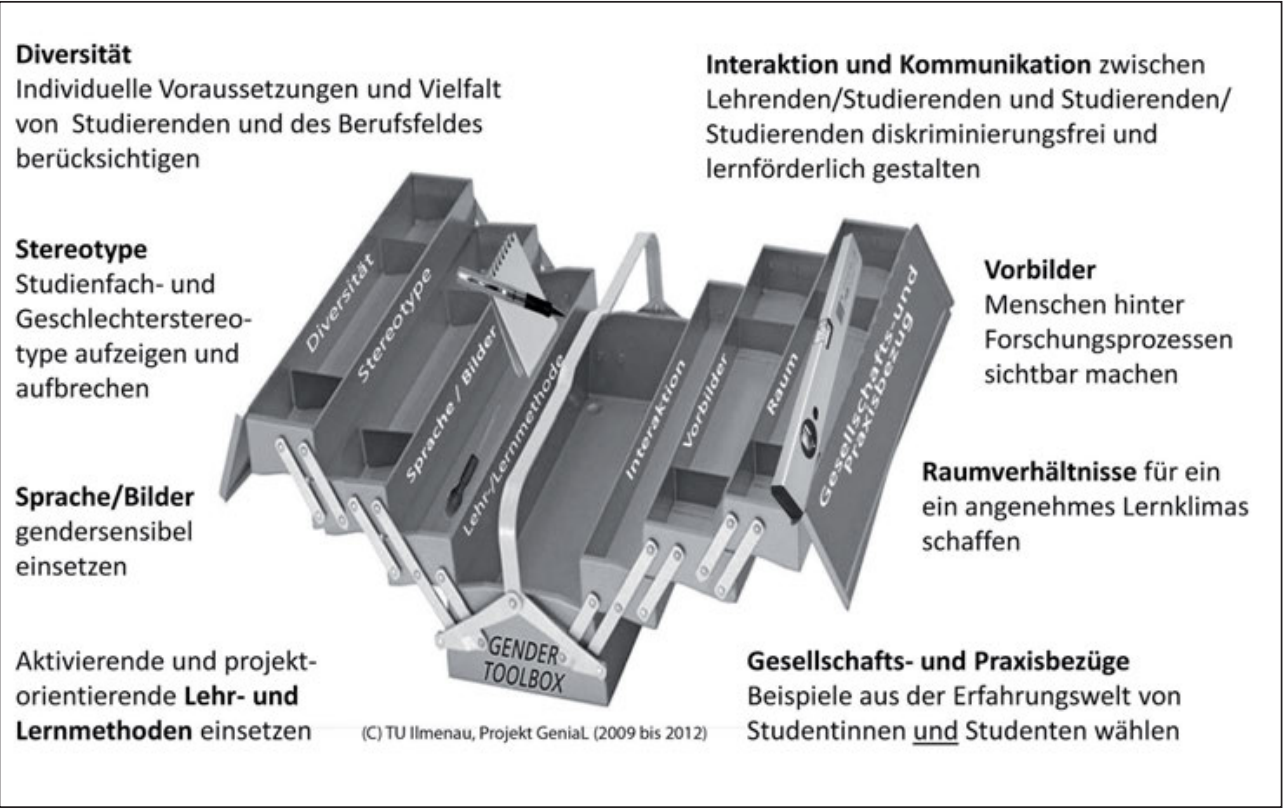

Abb. 1: $\quad$ Aufbau der Gender-Diversity-Toolbox

Das Konstrukt der Gender-Diversity-Toolbox ist flexibel zu betrachten, das heißt, die Dimensionen sind eng miteinander verknüpft, keinesfalls trennscharf und können entsprechend der eigenen Lehranforderungen und Lehrbedürfnisse modifiziert und weiterentwickelt werden. Die folgende Ausführung soll die Funktionsweise der Gender-Diversity-Toolbox verdeutlichen und bezieht sich dabei auf die Dimensionen „Stereotype“, „Sprache/Bilder“ und „Vorbilder".

\section{Ausgangsproblem:}

Trotz der verhältnismäßig jungen Geschichte der Informatik wurden, wie in vielen anderen Bereichen der Technikentwicklung und Technikgestaltung auch, die Beiträge und Erfolge von Frauen nicht ausreichend sichtbar gemacht. Dies birgt die Gefahr einer biologisierten, geschlechterpolarisierenden und stereotypisierten Zuschreibung: Frauen wären von Natur aus technikdistanziert, Männer hingegen technikaffin (Hausmann und Hettich, 1995).

Empfehlungen der Gender-Diversity-Toolbox:

1. Machen Sie Personen hinter den Forschungsprozessen sichtbar!

2. Recherchieren Sie nach historischen und/oder aktuellen Beiträgen von Informatikerinnen und Informatikern!

3. Entkräften Sie Biologismen durch empirisch belastbare Kenndaten! So liegt beispielsweise dem Global Education Digist (2009) zu Folge der Frauenanteil in MINTStudiengängen mit $45 \%$ in der Mongolei, $36 \%$ in Kolumbien und $35 \%$ in Bulgarien höher als in Deutschland (UNESCO, 2009).

Eine sehr homogene Fachkultur kann eine Vielzahl weiterer Stereotypisierungen und Klischees produzieren und/oder verfestigen. Hierfür hält die Gender-Diversity-Toolbox ebenfalls Empfehlungen bereit: 
Thematisieren, diskutieren und hinterfragen Sie gemeinsam mit Ihren Studierenden medialisierte Pauschalisierungen und klischeehafte Darstellungen:

1. Nationalstereotype (z. B. Computer-Inder, Software-Republik Korea)

2. Altersstereotype (z. B. technikaffine Jugend vs. technikdistanzierte Ältere)

3. Fachgruppenstereotype (z. B. Konnotation eines Informatikers als männlich, unsozialer und unkommunikativer Nerd)

Die vielfältigen Themen- und Einsatzbereiche der Informatik (z. B. Kommunikation, Gesundheit, Kultur etc.) und ihre starke Durchdringung des gesellschaftlichen Alltags, erfordern zunehmende Kenntnisse der User-Vielfalt. So können heterogene Teambesetzung, reflexives Gender- und Diversity-Wissen sowie Usability-Tests im Rahmen der Technikentwicklung einen wichtigen Beitrag dazu leisten, zielgruppenspezifische Bedürfnisse zu erfassen (z. B. Senioren-Handy mit extra großen Tasten und vereinfachter Menüführung) und die mit klischeehaften Vorstellungen verbundenen Fehlproduktionen zu vermeiden (z. B. PuderdosenHandy für Frauen). Einen praktischen Leitfaden zur Ermittlung von Gender-Aspekten bei der Technikgestaltung hat die Fraunhofer Gesellschaft im Rahmen ihres Projektes „Discover Gender“ zusammengestellt (Döring et al., 2013; John et al., 2012). Der Leitfaden ist abrufbar unter URL: www.ffg.at/getdownload.php?id=3138 (Abgerufen am 21.10.2013). Für nähere Informationen zum Projekt „Discover Gender“ siehe Bessing und Lukoschat (2007).

Empfehlungen der Gender-Diversity-Toolbox:

1. Berücksichtigen Sie auch Beiträge von Personen anderer Wissenschaftsdisziplinen, die einen Beitrag zur Informatik geleistet haben bzw. leisten!

2. Zeigen Sie die Vielfalt des späteren Berufsfeldes und eines damit verbundenen breiten Aufgabenspektrums auf (z. B. Beratungs- und Dienstleistungssektor mit starker Kundenorientierung und Arbeit in interdisziplinären und interkulturellen Teams)!

3. Verdeutlichen Sie die Relevanz einer zielgruppenorientierten Technikgestaltung sowie Notwendigkeit und Mehrwehrt von Usability-Tests bereits während der Entwicklungsphase!

4. Sensibilisieren Sie Ihre Studierenden für die Relevanz, bei der Technikentwicklung, Technikgestaltung sowie Techniknutzung, das Knowhow und Untersuchungsmethoden anderer Fachdisziplinen (z. B. sozial- und wirtschaftswissenschaftliche Disziplinen) einzubeziehen!

5. Diskutieren Sie technische Entwicklungen mitunter auch kontrovers (z. B. Abwägen von Nutzen und Risiken für die jeweiligen Usergruppen)!

\section{Gender/Diversitytool für Informatik-Lehre an der Fachhochschule Erfurt}

Im Rahmen des Projektes GeniaL wurde unter Verwendung der Gender-Diversity-Toolbox an der Fachhochschule Erfurt ein Tool für die Lehrfächer Software-Ergonomie und WebUsability entwickelt. Studierende sollen sich hierbei mit folgenden Fragestellungen auseinandersetzen:

- Was bedeutet Gender in der Informatik?

- Wirkt Gender im Softwareentwicklungsprozess?

- Zeigen sich Gender-Einflüsse in Softwareprodukten? 
Nach einer kurzen Einführungs- und Motivationssequenz - „Warum beschäftigen wir uns überhaupt damit?" - wird den Studierenden anhand von Software- und UsabilityEngineering-Vorgehensweisen erläutert, wie im Softwareentwicklungsprozess Einflüsse von Gender sowohl bei der Entwicklung als auch bei der Nutzung von Produkten eine Rolle spielen. Dabei werden die Studierenden angehalten, über nachfolgende Fragen (Bath, 2008) nachzudenken:

- Gibt es eine Abwesenheit von Geschlechterverhältnissen in der Informatik?

- Werden Ausschlüsse bereits bei der Aufgabenanalyse der Softwareentwicklung produziert?

- Finden Rückgriffe auf geschlechtsstereotypische Grundannahmen statt?

- Wird geschlechtsspezifische Arbeitsteilung durch Informatik verstärkt?

Dazu beschäftigen sich die Studierenden in einer Übung mit vorgegebenen Beispielen (auch mit geschlechtsstereotypischen Übertreibungen) und gehen dabei den Fragen nach

- Wie zeigen sich Differenz und Diversität bzgl. Gender bei der Softwareentwicklung und den Softwareprodukten?

- Was würden Sie warum im Prozess der Entwicklung diesbezüglich tun?

- Worauf achten Sie dafür bei Softwareprodukten?

Die Studierenden arbeiten in Gruppen und präsentieren anschließend ihre Erkenntnisse den anderen Kursteilnehmerinnen und Kursteilnehmern. Abschließend werden ihre Ergebnisse dann gemeinsam und mit Hilfe der Professorin abstrahiert und eingeordnet.

Das Tool wurde im letzten Sommersemester getestet und zeigte, dass die Studierenden eine zielgruppenorientierte Softwareentwicklung für wichtig erachteten, für das Thema Gender aber bisher wenig sensibilisiert waren. Dies betraf übrigens sowohl Studentinnen als auch Studenten. Eine erneute Testphase steht im Sommersemester 2014 an, über die Ergebnisse informieren wir bei Interesse.

\section{Gendertool für Informatik-Lehre an der Technischen Universität Ilmenau}

An der Technischen Universität Ilmenau kam die Gender-Diversity-Toolbox in InformatikVorlesungen des Grundlagenstudiums für Studierende des 1. bis 4. Fachsemesters zum Einsatz. Im Folgenden werden einige Beispiele genannt, die von Studierenden positiv bewertet wurden.

Beispiel 1:

Frühzeitige Einbindung von Studentinnen und Studenten in karrierefördernde Netzwerke

- Machen Sie Ihre Studierenden auf wichtige Netzwerke und Fachcommunties (z. B. Youngnet des VDE - Verband der Elektrotechnik, Elektronik, Informationstechnik e. V. oder die Fachgruppe GI - Frauen der Gesellschaft für Informatik e. V.) sowie Veranstaltungen (,informatiCup“) aufmerksam und ermuntern Sie sie zur Teilnahme! Hinweis: „,informatiCup“ ist ein jährlicher Wettbewerb, der von der Gesellschaft für Informatik (GI) für Studierende der Informatik ausgerichtet wird. 
Beispiel 2:

Weiterführende Informationen und interdisziplinäre Bezüge

- Binden Sie weiterführende Informationen zum Thema/Forschungsfeld mit ein (z. B. Statistiken über zukünftige Arbeitsfelder, aktuelle Forschungsthemen der/des Lehrenden etc.), die Sie über eine Lehrfolie oder alternativ über Vorlesungsskripte, Newsletter, Info-Wand oder Internetseite des Fachgebietes kommunizieren!

\section{Beispiel 3:}

Aufzeigen von Personen hinter Forschungsprozessen sowie vorbildhafte Frauen und Männer im Bereich der Informatik

- Nutzen Sie gut aufbereitete Online-Datenbanken (z. B. www.fembio.org oder www.frauen-informatik-geschichte.de) und Publikationen (z. B. „Zukunft gestalten: Ich werde Informatikerin!“ vom Kompetenzzentrum Technik-Diversity-Chancengleichheit e. V.) als Recherchegrundlage!

- Ziehen Sie auch Personen aus Ihrem eigenen Arbeitsumfeld (z. B. Forschungsteam/Fachgebiet/Institut/Hochschule) heran!

Beispiel 4:

Aufzeigen der gesellschaftlichen Bedeutung von Informatik

- Verdeutlichen Sie die gesellschaftliche Relevanz von Informations- und Kommunikationstechnologien in unserem Alltag!

- Animieren Sie Ihre Studierenden zu weiterführenden Fragestellungen (z. B. Integrationsfunktion von Technik am Beispiel der Internetnutzung von Menschen mit visuellen Handicaps; Gestaltungskritierien für ein barrierefreies Internet etc.)!

Bei den hier gezeigten Beispielen der Gender-Diversity-Toolbox ist zu beachten, dass es sich um niedrigschwellige und punktuelle Angebote handelt. Sie verhelfen insbesondere Lehrenden ohne spezifische Gender- und Diversity-Kenntnisse zu einem ersten Zugang, können aber mitunter auch zur eigenen, vertiefenden Auseinandersetzung mit Gender und Diversity motivieren. Darüber hinaus eignen sich die Dimensionen der Gender-Diversity-Toolbox auch gut dazu, die eigene Lehre auf Gender- und Diversity-Aspekte zu überprüfen.

\section{$4 \quad$ Fazit}

Bei den hier vorgestellten Beispielen handelt es sich um einen ersten integrativen Ansatz zur Gestaltung gender- und diversitysensibler Informatik-Veranstaltungen im Grundlagenstudium der Fachhochschule Erfurt und der Technischen Universität Ilmenau. Nach den bisherigen Erfahrungen eignen sich diese Maßnahmen als Türöffner, um Lehrende und Studierende der Informatik für Gender- und Diversity-Themen zu sensibilisieren. Mit Hilfe der Gender- 
Diversity-Toolbox können Informatik-Lehrende, auch ohne große Einarbeitungszeit in sozialwissenschaftliche Theorien, gender- und diversitysensible Lehrmaterialien konzipieren.

Für Studierende stellt die Berücksichtigung von Gender- und Diversity-Aspekten im Rahmen der Lehrveranstaltung einen Mehrgewinn dar, da ihre individuellen Interessen und Bedürfnisse im Lehr-/Lernprozess eine stärkere Beachtung finden. Darüber hinaus bereitet sie die inhaltliche Auseinandersetzung mit gender- und diversityspezifischen Fragestellungen in der Informatik auf ihr späteres Berufsfeld vor (z. B. kunden- und zielgruppenorientiert Technikentwicklung).

Zur Förderung der reflexiven Gender-Diversity-Kompetenz und für eine tiefgreifende und langfristige Veränderung der Lehre und Fachkultur bedarf es jedoch umfassenderer Beratungsmaßnahmen und Weiterbildungsangebote, sowohl für Lehrende und Forschende als auch für Studierende. Zur Grundsensibilisierung von Lehrenden und Forschenden bieten sich hierbei Workshops an, die den Lehrenden in einem ersten Schritt Grundlagen zur Stereotypenforschung (Geschlecht, Alter, Fachkultur etc.) und zentrale Ansätze der GenderDiversity-Forschung vermitteln. Darauf aufbauend werden dann in einem zweiten Schritt die Lehrenden dazu befähigt, unter Berücksichtigung ihrer jeweiligen fachspezifischen Eigenheiten und hochschuldidaktischen Anforderungen an Informatik-Lehre, eigene gender- und diversitysensible Lehrmaterialien zu entwickeln. Diese sollen sich in den bestehenden Lehrstoff integrieren lassen. Für Forschende der Informatik bietet sich als Aufbaukurs eine Praxiswerkstatt an, in der sie beispielsweise gemeinsam mit Forschender anderer Fachdisziplinen Projektideen generieren.

Darüber hinaus bieten Beratungs- und Coaching-Angebote die Möglichkeit, InformatikLehrende und Forschende durch gender- und diversitygeschulte Hochschuldidaktikerinnen und Hochschuldidaktiker individuell über einen längeren Zeitraum zu begleiten. Allerdings gilt es bei diesem Vorgehen, den entsprechend höheren Zeit- und Personalaufwand zu beachten.

Für Studierende wurde an der Technischen Universität Ilmenau der fachübergreifende Grundlagenkurs „Gender- und Diversity im Berufs- und Privatleben“ entwickelt, der aufgrund seines breiten Themenspektrums (z. B. Identität und Sexualität, Familie und Paarbeziehungen, Medien, Sport, Beruf, Technik etc.) die gesellschaftliche Relevanz von Gender und Diversity aufzeigt. Im Hinblick auf die Informatik wird hier unter anderem die im Beispiel 4 dieses Artikels angeführte Integrationsfunkton von Technik (IT-Nutzung von Menschen mit visuellen und akustischen Handicaps; Gestaltungskriterien für ein barrierefreies Internet etc.) beleuchtet. Aufgrund des großen Interesses bei den Teilnehmenden wurde ein weiterführender Kurs entwickelt (z. B. Musik, Ernährung, Politik etc.). Beide Kurse werden als Online-Kurs angeboten und sind im Lehrangebot des Studium Generale verankert.

Zukünftig gilt es die hier vorgestellten Maßnahmen und Beispiele zu erweitern und insbesondere die Reflexions-, Interaktions- sowie Diskursprozesse von Lehrenden und Studierenden im Hinblick auf Gender und Diversity stärker zu fördern und die Bewertung dieser Angebote durch die Teilnehmenden zu erfassen. Im Rahmen des Thüringer Kompetenznetzwerks Gleichstellung (TKG) spielen daher in der Verbundarbeit der Thüringer Hochschulen Konzeption, Umsetzung und Evaluation hochschuldidaktischer (Weiter-)Bildungsangebote zu Gender und Diversity eine zentrale Rolle. 


\section{Literatur}

Augustin, S. \& Probstmeyer, K. (2013). Gender in der akademischen Lehre an Thüringer Hochschulen: Praktische Beispiele für MINT-Studiengänge. In N. Hille \& B. Unteutsch (Hrsg.), Gender in der Lehre. Best-Practice-Beispiele für die Hochschule (S. 103-110). Opladen: Westdeutscher Verlag.

Bath, C. (2008). De-Gendering von Gegenständen der Informatik: Ein Ansatz zur Verankerung von Geschlechterforschung in der Disziplin. In B. Schwarze, M. David \& B. C. Belker, (Hrsg.), Gender und Diversity in den Ingenieurwissenschaften und der Informatik (S. 166-182). Bielefeld: UVW.

Becker, R., Jansen-Schulz, B., Kortendiek, B. \& Schäfer, G. (Hrsg.) (2001), Gender-Aspekte bei der Einführung und Akkreditierung gestufter Studiengänge - eine Handreichung. Studien Netzwerk Frauenforschung, 21, NRW.

Beier, M. \& von Gizycki, V. (Hrsg.) (2002). Usability, Nutzerfreundliches Web-Design. Berlin [u.a.]: Springer.

Berszinski, S., Nikoleyczik, K., Remmele, B., RuizBen, E., Schinzel, B., Schmitz, S. \& Stingl, B. (2002). Geschlecht (SexGender): Geschlechterforschung in der Informatik und an ihren Schnittstellen. FifF-Kommunikation, 3, 32-37.

Bessing, N. \& Lukoschat, H. (2007). Gender und Innovationen - Erfahrungen aus dem Projekt „Discover Gender!“”. In C. Leicht-Scholten (Hrsg.), Gender and Sciences. Perspektiven in den Natur- und Technikwissenschaften (S. 69-82). Bielefeld: transcript.

Derboven, W. \& Winker, G. (2010). Ingenieurwissenschaftliche Studiengänge attraktiver gestalten. Vorschläge für Hochschulen, Berlin [u.a.]: Springer.

Döring, N. \& Probstmeyer, K. (im Druck). Gender-Diversity-Tools für Lehrende im ingenieurwissenschaftlichen Grundlagenstudium: Ein praxiserprobter Werkzeugkasten. diversitas - Zeitschrift für Managing Diversity und Diversity Studies.

Döring, N., Augustin, S. \& Probstmeyer, K. (Hrsg.) (2013). Gender in der akademischen Lehre an Thüringer Hochschulen (GeniaL). Ausgewählte Forschungsergebnisse des Thüringer Verbundprojektes (Projektlaufzeit 2009 bis 2012). Langewiesen: Ilmprint.

Erharter, D. (2012). Gendability - Was hat Usability mit Gender zu tun? In G. Kempter, K.H.Weidmann (Hrsg.), Technik für Menschen im nächsten Jahrzehnt. Beiträge zum Usability Day X (S. 107-117). Lengerich : Pabst Science Publ.

Engler, S. (1993). Fachkultur, Geschlecht und Soziale Reproduktion. Eine Untersuchung über Studentinnen und Studenten der Erziehungswissenschaft, Rechtswissenschaft, Elektrotechnik und des Maschinenbaus. Weinheim: Deutscher Studienverlag.

Gesellschaft für Informatik e.V., Bedeutende Informatikpersönlichkeiten. Abgerufen am 12. Oktober 2013 von http://www.gi.de/service/downloads.html

Goldmann, O.: Frauen in der Informatik. Abgerufen am 12. Oktober 2013 von http://www.virtosphere.de/schillo/teaching/WS2001/Vortraege/Frauen.ppt

Götsch, Monika (2013). „Das fängt natürlich an mit irgendwelchen Spielekonsolen“ - oder: Was dazu motiviert, Informatik (nicht) zu studieren. Informatik-Spektrum, 36 (3), 331-338.

Grünewald, E. \& von Gunten, A. (2009). Werkmappe Genderkompetenz. Materialien für geschlechtergerechtes Unterrichten. Zürich: Verlag Pestalozzianum.

Hausmann, M. \& Hettich, C. (1995). Geschlechterunterschiede beim Zugang zu Neuen Technologien. Tübingen: Universitätsverlag.

Jansen-Schulz, B. \& van Riesen, K. (2009). Integratives Gendering in Curricula, Hochschuldidaktik und Aktionsfelder der Leuphana Universität Lüneburg. In N. Auferkorte-Michaelis, I. Stahr, A. Schön- 
born \& I. Fitzek (Hrsg.), Gender als Indikator für gute Lehre: Erkenntnisse, Konzepte und Ideen für die Hochschule (S. 65-86). Opladen [u.a.]: Budrich UniPress.

John, A., Probstmeyer, K., Saarmann, S. \& Schade, G. (2012). Gender in der akademischen Lehre ausgewählte Ergebnisse für die Lehrpraxis in der Informatik. Frauen machen Informatik, 36, 22-25.

Liebig, B., Rosenkranz-Fallegger, E. \& Meyerhofer, U. (Hrsg.) (2009). Handbuch Genderkompetenz. Ein Praxisleitfaden für (Fach-) Hochschulen. Zürich: vdf Hochschulverlag.

Münst, A. S. (2002). Wissensvermittlung und Geschlechterkonstruktionen in der Hochschullehre: Ein ehtnographischer Blick auf natur- und ingenieurwissenschaftliche Studienfächer. Weinheim: Deutscher Studien-Verlag.

Probstmeyer, K., Döring, N. \& Augustin, S. (2011). Geschlechtersensibilität im ingenieurwissenschaftlichen Grundlagenstudium. In H. Hanno, S. Kersten \& M. Köhler (Hrsg.), Renaissance der Ingenieurpädagogik. Entwicklungslinien im Europäischen Raum (S. 124-132). Dresden: Verlag der Technischen Universität Dresden.

Ripke, M. \& Siegeris, J. (2011). Informatik - ein Männerfach!? Monoedukative Lehre als Alternative. Informatik-Spektrum, 35(5), 331-338.

Roloff, C. (1998). Frauen in natur- und ingenieurwissenschaftlichen Studiengängen - Problembeschreibung am Beispiel von Chemie- und Informatik-Studentinnen. In D. Janshen \& H. Rudolph (Hrsg.), Frauen gestalten Technik. Ingenieurinnen im internationalen Vergleich (S. 32-40). Pfaffenweiler: Centaurus-Verlagsgesellschaft.

Rosser, S. V. (1998). Teaching the Majority. Breaking the gender barrier in science, mathematics, and engineering. New York: Teachers College Press.

Schinzel, B. (2012). Geschlechtergerechte Informatik-Ausbildung an Universitäten. In M. Kampshoff \& C. Wiepcke (Hrsg.), Handbuch Geschlechterforschung und Fachdidaktik (S. 331-344). Wiesbaden: Verlag für Sozialwissenschaften.

VDI-monitorING. Daten zu Arbeitsmarkt, Hochschule und Schule. Abgerufen am 22. Oktober 2013 von http://www.vdi.de/wirtschaft-politik/arbeitsmarkt/monitoring-datenbank 